\title{
BMJ Open Impact on Mental, Physical and Cognitive functioning of a Critical care sTay during the COVID-19 pandemic (IMPACCT COVID-19): protocol for a prospective, multicentre, mixed- methods cohort study
}

\author{
Ana Cristina Castro-Ávila (D) ,1,2 Catalina Merino-Osorio (D) ,1 \\ Felipe González-Seguel (D) ,1,3 Agustín Camus-Molina (D) ,1,3 Jaime Leppe (D) , ${ }^{1}$ The \\ IMPACCT COVID-19 study group
}

To cite: Castro-Ávila AC, Merino-Osorio C, GonzálezSeguel F, et al. Impact on Mental, Physical and Cognitive functioning of a Critical care sTay during the COVID-19 pandemic (IMPACCT COVID-19): protocol for a prospective, multicentre, mixed-methods cohort study. BMJ Open 2021;11:e053610. doi:10.1136/ bmjopen-2021-053610

- Prepublication history and additional supplemental material for this paper are available online. To view these files, please visit the journal online (http://dx.doi.org/10.1136/ bmjopen-2021-053610).

Received 18 May 2021 Accepted 19 August 2021

Check for updates

(c) Author(s) (or their employer(s)) 2021. Re-use permitted under CC BY-NC. No commercial re-use. See rights and permissions. Published by BMJ.

For numbered affiliations see end of article.

Correspondence to Mr Felipe González-Seguel; feligonzalezs@udd.cl

\section{ABSTRACT}

Introduction The ongoing pandemic could affect the duration, variety and severity of the mental, physical, and cognitive impairments intensive care unit (ICU) survivors and their families frequently present. We aim to determine the impact of the COVID-19 pandemic on the mental, physical, and cognitive health of survivors, the experience of their families and their treating healthcare professionals.

Methods and analysis Prospective, multicentre, mixed-methods cohort study in seven Chilean ICUs. Sample: 450 adults, able to walk independently prior to admission, in ICU and mechanical ventilation $>48$ hours with and without COVID-19. Clinical Frailty Scale, Charlson comorbidity index, mobility (Functional Status Score for the Status Score for the Intensive Care Unit) and muscle strength (Medical Research Council Sum Score) will be assessed at ICU discharge. Cognitive functioning (Montreal Cognitive Assessment-blind), anxiety and depression (Hospital Anxiety and Depression Scale), post-traumatic stress (Impact of Event Scale-Revised) symptoms, disability (WHO Disability Assessment Schedule 2.0), quality of life (European Quality of Life Health Questionnaire), employment and survival will be assessed at ICU discharge, 3 months and 6 months. A sample will be assessed using actigraphy and the Global Physical Activity Questionnaire at 6 months after ICU discharge. Trajectories of mental, physical, and cognitive impairments will be estimated using multilevel longitudinal modelling. A sensitivity analysis using multiple imputations will be performed to account for missing data and loss-to-followup. Survival will be analysed using Kaplan-Meier curves. The perceptions of family members regarding the ICU stay and the later recovery will be explored 3 months after discharge. Healthcare professionals will be invited to discuss the challenges faced during the pandemic using semistructured interviews. Interviews will be thematically analysed by two independent coders to identify the main themes of the experience of family members and healthcare professionals.
Strengths and limitations of this study

This is the first Chilean multicentre study assessing postintensive care unit (ICU) sequalae.

- Experiences of family members and healthcare professionals during the pandemic will also explored.

- Due to infection control protocols and lockdowns, most physical functioning measures are self-reported.

- Patient's ICU assessments were limited by increased workload and restrictions in access to the ICUs.

Ethics and dissemination The study was approved by the Clinica Alemana Universidad del Desarrollo Ethics Committee (2020-78) and each participating site. Study findings will be published in peer-reviewed journals and disseminated through social media and conference meetings.

Trial registration number NCT04979897.

\section{INTRODUCTION}

Postintensive care syndrome (PICS) is a common consequence of an intensive care unit (ICU) stay and can last up to 5 years. ${ }^{1-3}$ The family members are often affected, reporting diminished quality of life and mental health-related quality of life. ${ }^{4}$ About $80 \%$ of family members become informal carers, and $33 \%$ of families see a significant reduction of income the first 6 months after discharge. ${ }^{5}$ The extent to which these problems will be modified by the COVID-19 pandemic remains unknown.

If under normal circumstances, an ICU stay has detrimental effects, the pandemic added three extra factors. First, a rapid and exponential increase in acute care bed capacity might 
have affected the quality of care delivered by spreading too thin highly skilled healthcare staff. Second, COVID-19 involves a new disease with great uncertainties regarding treatment, prognosis and long-term effects. Early reports suggest that $64 \%$ of patients who were discharged from ICU after COVID-19 have at least one symptom of PICS at 6 months after discharge ${ }^{6}$ and $32 \%$ had anxiety or depression symptoms, ${ }^{7}$ which suggests these patients will have similar impairments to what has been reported for other ICU survivors previously. ${ }^{89}$ Third, infection control protocols meant that healthcare staff had to wear personal protective equipment, and family visiting was restricted. ${ }^{10}$ These factors add another layer of potential negative effects due to challenges in communication with patients and their family members. Therefore, we hypothesise that the prevalence and severity of mental, physical and cognitive impairments will be higher in patients treated in periods of higher bed occupancy and those who had COVID-19. In the case of family members, we expect that the experience of having a next of kin in the ICU during the pandemic would be stressful and traumatic, but those with social support will cope better as the stress process model suggests. ${ }^{11}$ In the case of staff members, their experiences will vary greatly depending on their profession and workplace, but we expect places with a more open/ less hierarchical structure to have coped better with the increase in demand because they adapt faster to change. ${ }^{12}$

The primary objective of this study is to compare the trajectory of mental, physical and cognitive impairments at ICU discharge, 3 and 6 months of mechanically ventilated adult patients who survived an ICU stay due to COVID-19 or other causes during high and low bed occupancy in the pandemic. Secondary objectives are:

- To compare the employment status, quality of life and survival rate at ICU discharge, 3 months and 6 months of patients who were admitted to ICU due to COVID-19 or other causes during high and low bed occupancy in the pandemic.

- To describe the sedentary behaviours and physical activity levels in a sample of ICU survivors during the COVID-19 pandemic using a 1-week actigraphy protocol.

- To explore the psychological and emotional experiences reported by family members/next of kin of patients admitted to the ICU during the COVID-19 pandemic.

- To explore the emotional, intellectual, physical and administrative challenges faced by the participating ICU staff during the COVID-19 pandemic.

- To evaluate the feasibility of the follow-up from ICU discharge to 3 months and 6 months during the pandemic.

\section{METHODS}

\section{Study design and setting}

The 'Impact on Mental, Physical, And Cognitive functioning of a Critical care sTay during the COVID-19 pandemic' (IMPACCT COVID-19) is a prospective, multicentre, cohort study in seven Chilean academic medical-surgical ICUs. This study also involves a qualitative component including semistructured interviews with family members/next of kin of ICU survivors and with ICU staff from the participating centres. Participating sites are four public and three private hospitals comprising a pooled bed capacity of about 200 ICU beds for both patients with COVID-19 and patients admitted for other causes. The IMPACCT COVID-19 study started in October 2020, and the initial recruitment at ICU discharge has ended. Data collection is planned until November 2021 to achieve completion of the study in February 2022.

\section{Study population and eligibility criteria}

Within 72 hours after ICU discharge, adult patients ( $\geq 18$ years old) who are mechanically ventilated for at least 48 hours in one of the participating ICUs and do not meet any of the exclusion criteria (table 1 ) will be invited to participate.

\section{Procedure}

The planned flow of participants throughout the study is presented in figure 1. Patients will be screened daily to identify those that are in conditions for ICU discharge. Each site coordinator, which is a clinician physiotherapist responsible for the site, will check that the patient is delirium free (CAM-ICU negative) and cooperative (ie, using five standardised questions: open (close) your eyes; look at me; open your mouth and stick out your tongue; nod your head; and raise your eyebrows when I have counted up to five ${ }^{13}$ ) within 72 hours from ICU discharge. Every patient deemed eligible will be invited to participate through a face-to-face visit by the assigned evaluator, receiving verbal and written information about the study. Patients will be assessed at ICU discharge (T1; defined by the point between medical decision of discharge until 72 hours after), 3 months (T2) and 6 months after ICU discharge (T3). Fifty-eight physiotherapists were trained for the assessments at ICU discharge, which included in-person measurements and self-administered questionnaires. Physiotherapists had to be working in one of the participating ICUs at the time of the training. Training for standardising $\mathrm{T} 1$ assessments was delivered by experienced physiotherapists and researchers (AC-M, CM-O and FG-S). For the follow-up assessments (T2 and T3), patients will be contacted via email or telephone to schedule a phone call evaluation performed by trained interviewers. Additionally, physical activity and sedentary behaviours will be assessed using actigraphy 6 months after ICU discharge in a sample of participants (details described further).

After the patient agreed to participate and signed the informed consent form, the following baseline data will be collected from the patient clinical records: age, gender, body mass index, highest educational level achieved (no formal education, primary school, secondary school, undergraduate or postgraduate), admission diagnosis, 
Table 1 Exclusion and stopping follow-up criteria

\begin{tabular}{ll} 
Exclusion criteria & Rationale \\
\hline Unable to walk independently 2 weeks prior to ICU admission (with or without a gait aid) & Potential confounding factor \\
S5q<5 or CAM-ICU positive within 72 hours after ICU discharge & Unable to evaluate \\
Patient who do not understand or speak Spanish & Unable to evaluate \\
Patient unable to communicate verbally & Incomplete assessment data \\
Burn or severe trauma as admission diagnosis & Incomplete assessment data \\
$\begin{array}{l}\text { Any neurological disorder (ie, spinal cord injury, stroke and brain tumours) as admission } \\
\text { diagnosis }\end{array}$ & Potential confounding factor \\
Transferred to a non-participating study centre before ICU discharge assessment & Unable to evaluate \\
Recent prolonged hospital stay (extended by more than 3 months) & Potential confounding factor \\
Criteria to stop follow-up & Potential confounding factor \\
Readmission after being ICU discharged & Incomplete assessment data \\
Withdrawal of consent & Incomplete assessment data \\
\hline Death before 3 months or 6 months from ICU discharge &
\end{tabular}

CAM-ICU, confusion assessment method for the intensive care unit; ICU, intensive care unit; s5q, standardised five questions.

Charlson Comorbidity Index, duration of mechanical ventilation, length of hospital stay before ICU admission, ICU length of stay, number of intubations, and the maximum level of organ system support received. ${ }^{14}$

\section{Measurement outcomes}

The assessment points and measurement instruments are presented in table 2. Measurement instruments were selected according to the recommended Core Outcome Measurement Set for critical illness survivors. ${ }^{1516}$

When available, we used the Chilean version of each instrument, otherwise, the validated version in Spanish. Trained physiotherapists will take an estimated maximum time of $70 \mathrm{~min}$ to perform the assessment at ICU discharge (T1). A trained interviewer will take an estimated maximum time of $20 \mathrm{~min}$ to apply the questionnaires by telephone at 3 months (T2) and 6 months (T3) after ICU discharge.

The primary outcome measure is disability assessed at 6 months after ICU discharge using the WHO Disability Assessment Schedule 2.0 (WHODAS 2.0), which is recommended for critical illness survivors. ${ }^{8}$ The WHODAS 2.0 is a self-reported disability questionnaire based on the International Classification of Functioning, Disability, and Health. It includes 36 questions, organised under six domains (cognition, mobility, self-care, getting along, life activities, and participation). Each question must be answered based on the perceived difficulty for performing activities using a five-point scale (none, mild, moderate, severe, and extreme).${ }^{17}$ We will use the Spanish version freely available at https://appswhoint/ iris/handle/10665/170500. ${ }^{18}$ The estimated response time ranges from 5 to $10 \mathrm{~min}$ when evaluated in-person at ICU discharge and 10-20 min when evaluated by telephone at 3 months and 6 months after ICU discharge.
Secondary outcomes measures

Clinical Frailty Scale (CFS)

The Clinical Frailty Scale (CFS) is a clinical judgement based tool developed for the Canadian Study of Health and Aging to evaluate the degree of frailty in elderly patients. ${ }^{19}$ Currently, it is also used for critically ill patients. $^{20}$ The CFS evaluates specific domains including physical functioning, activities of daily living (ADLs), instrumental ADL, assistance for personal care, comorbidities, and cognition to generate a frailty score using a nine-point scale ranging from 1 (very fit) to 9 (terminally ill). A score greater than 4 is considered fragile. ${ }^{19}$ We will use the Spanish version and recommended training material by the developers at the Dalhousie University. ${ }^{1921}$ The estimated scoring time ranges from 1 to $5 \mathrm{~min}$ evaluated in-person at ICU discharge considering the status 2 weeks before the onset of symptoms.

\section{Medical Research Council Sum Score (MRC-SS)}

Limb muscle strength will be assessed using the MRC$\mathrm{SS}$, which consists in a standardised examination of six muscle groups bilaterally (ie, shoulder abduction, elbow flexion, wrist extension, hip flexion, knee extension, and dorsiflexion). ${ }^{22}$ All muscle groups are scored using a six-point scale between 0 and $5(0=$ no visible/ palpable contraction; $1=$ visible/palpable contraction or no limb movement; $2=$ limb movement, but not against gravity; $3=$ movement against the gravity over nearly the entire range of motion; $4=$ motion against gravity and resistance, subjectively adjusted for gender and age; and $5=$ normal force). This scale requires an estimated assessment time of 5-10 min and will be evaluated only at ICU discharge following the method described by Hermans et $a$. $^{23}$ 


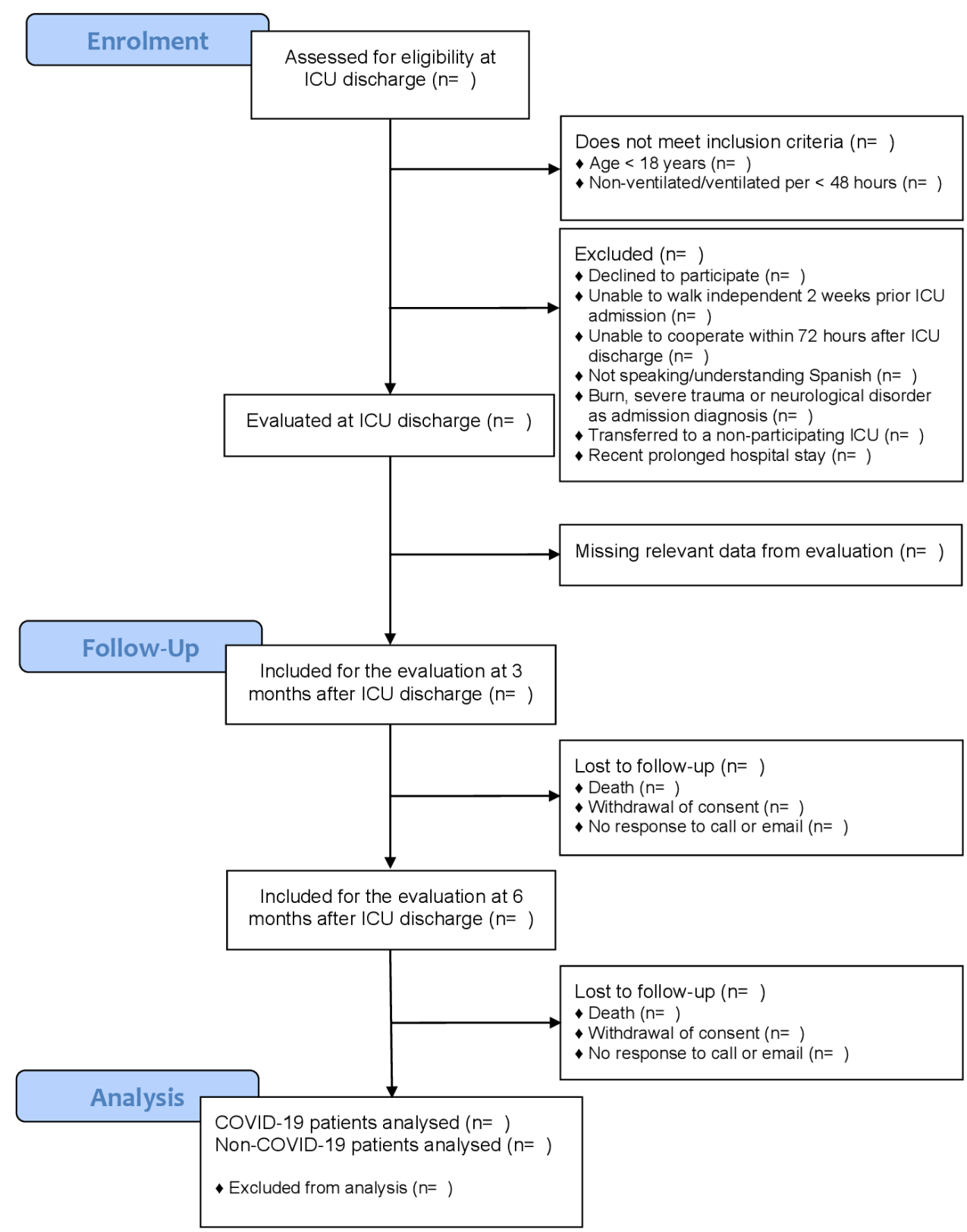

Figure 1 IMPACCT COVID-19 study flow chart. ICU, intensive care unit. IMAPCCT COVID-19, Impact on Mental, Physical, And Cognitive functioning of a Critical care sTay during the COVID-19 pandemic.

Functional Status Score for the Intensive Care Unit (FSS-ICU)

The FSS-ICU is a mobility instrument to score the level of physical assistance required when performing five functional activities: rolling, transfer from supine to sit, sitting at the edge of the bed, transfer from sitting to stand and walking. ${ }^{24}$ Each activity is scored using a seven-point scale ranging from 0 (not able to perform) to 7 (complete independence). The resulting overall score ranges from 0 to 35 points. Each evaluation requires between $10 \mathrm{~min}$ and $30 \mathrm{~min}$. It will be assessed at ICU discharge using the available and validated Chilean version. ${ }^{25}{ }^{26}$ Due to the limitations during the pandemic, walking will be evaluated inside the room, occasionally forcing the patient to walk with more laps than usual.
Montreal Cognitive Assessment-blind (MoCA-blind)

The MoCA-blind is a cognitive screening tool designed to detect cognitive dysfunction in five areas: memory, attention, language, abstraction, and orientation. It requires 5 min to be completed ${ }^{27}$ Each domain is scored separately for a total score ranging from 0 to 22 points. A score equal to or greater than 18 points is considered normal cognition. To minimise memory bias, the MoCA-blind will be assessed using V.7.1 at ICU discharge (in-person), V.7.2 at 3 months (by telephone) and V.7.3 at 6 months (by telephone). ${ }^{28} 29$ One evaluator (AC-M) received training and certification by Test MoCA Inc, and then trained the rest of the evaluators following the standardised procedure available at https://wwwmocatestorg. As recommended 
Table 2 Schedule of enrolment and follow-up of the IMPACCT COVID-19 study

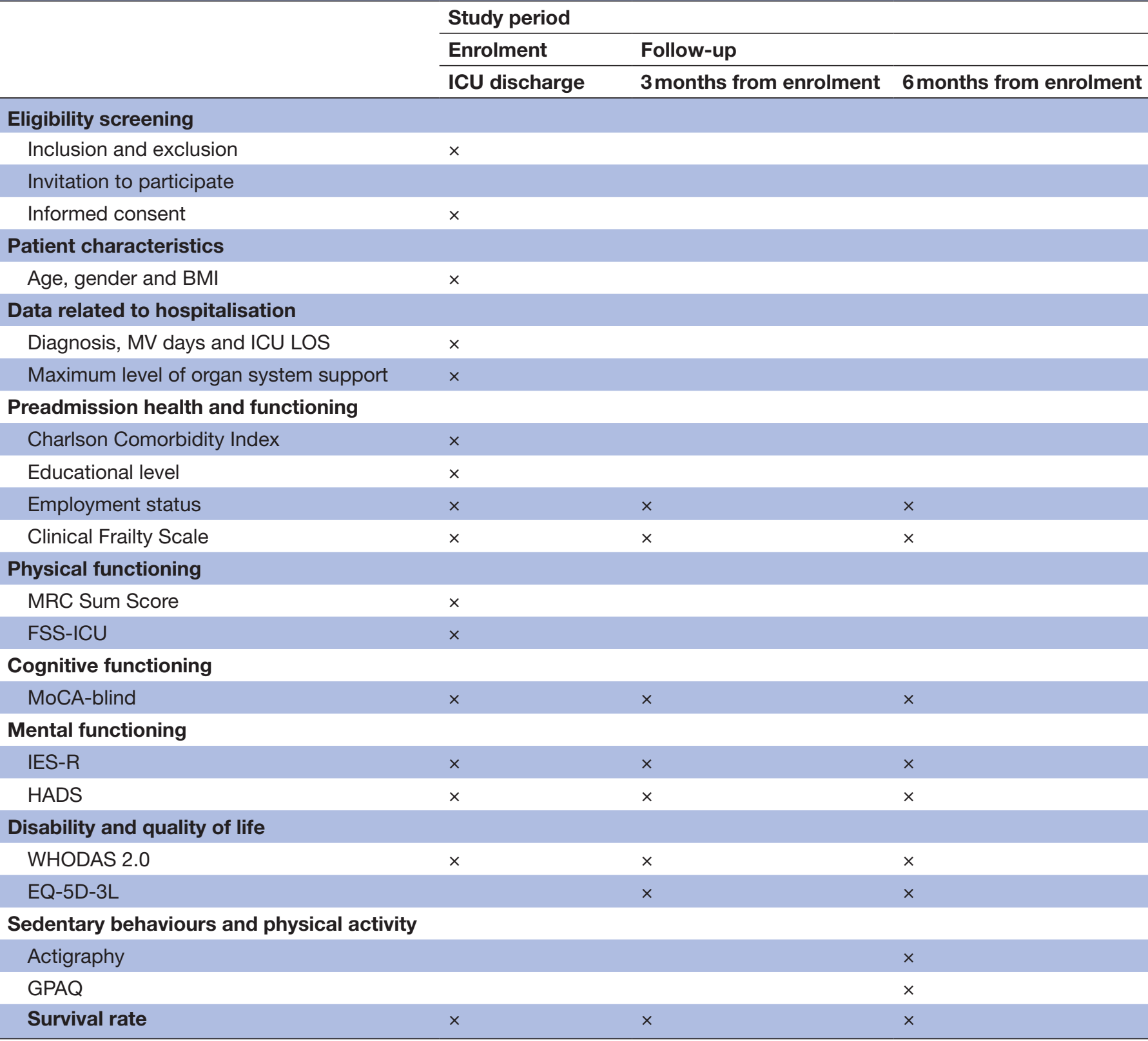

BMI, body mass index; IMPACCT COVID-19, Impact on Mental, Physical, And Cognitive functioning of a Critical care sTay during the COVID-19 pandemic; EQ-5D-3L, European Quality of Life Health Questionnaire 5 Domains; FSS-ICU, Functional Status Score for the Intensive Care Unit; GPAQ, Global Physical Activity Questionnaire; HADS, Hospital Anxiety and Depression Scale; ICU, intensive care unit; IES-R, Impact of Event Scale-Revised; LOS, length of stay; MoCA-blind, Montreal Cognitive Assessment-blind; MRC, Medical Research Council; MV, mechanical ventilation; WHODAS 2.0, WHO Disability Assessment Schedule 2.0.

by the developers, the results of this test will not be used for diagnostic purposes but as a cognitive screening.

Hospital Anxiety and Depression Scale (HADS)

The HADS is an interviewer or self-administered questionnaire designed to identify anxiety and depressive symptoms in a wide variety of in-hospital patients, which requires between 2 min and 5 min to be completed. ${ }^{30}$ The HADS has 15 questions-seven for anxiety and seven for depressive symptoms. Each question is rated with a fourpoint scale ranging from 0 ('absence') to 3 ('extreme presence'), resulting in a sum score of 21 points per subscale. HADS will be evaluated at ICU discharge and by telephone at 3 months and 6 months using the Chilean version. $^{31}$

Impact of Events Scale-Revised (IES-R)

The IES-R is an interviewer or self-administered questionnaire designed to measure the subjective distress caused by traumatic events that has been validated for critical illness survivors. ${ }^{32}$ It comprises 22 questions divided in three subscales: intrusion, avoidance, and hyperarousal. 
Questions are rated in a five-point scale ranging from 0 ('not at all') to 4 ('extremely'). The estimated response time is $6 \mathrm{~min}$. It will be evaluated at ICU discharge and by telephone at 3 months and 6 months using the available Chilean version. ${ }^{33}$

\section{European Quality of Life Health Questionnaire (EQ-5D-3L)}

The EQ-5D-3L is an interviewer or self-administered questionnaire of health status or health-related quality of life, including five domains: mobility, self-care, usual activities, pain/discomfort, anxiety/depression, and global health state. ${ }^{34}{ }^{35}$ Each domain is scored based on three levels of severity: no problems, some problems, and extreme problems. Additionally, EQ-5D-3L includes a visual analogue scale ranging from 'best imaginable health state' (100) to 'worst imaginable health state' $(0)$. Both parts of the questionnaire take an estimated response time of $2 \mathrm{~min}$. It will be evaluated by telephone at 3 months and 6 months using the Chilean version. ${ }^{36}$

\section{Employment status}

The employment status will be evaluated at ICU discharge, 3 months and 6 months using tailored questions regarding current occupation, working hours, and any changes to their employment situation as it has been used elsewhere. ${ }^{3738}$

\section{Survival}

The survival rate will be measured by the percentage of patients still alive at ICU discharge, 3 months and 6 months after ICU discharge. Information on deaths will be obtained from death certificates from the Chilean National Civil Registry.

\section{Sedentary behaviours and physical activity}

Sedentary behaviours and physical activity will be measured using a standardised 1 week actigraphy protocol according to the Chilean National Health Survey ${ }^{39}{ }^{40}$ using the ActiGraph GT3X (ActiGraph, Pensacola, Florida, USA) accelerometer and the Global Physical Activity Questionnaire. We will invite all participants at the 3-month follow-up phone call. Among those who agree to participate, we will recruit participants to achieve maximum variation in terms of age, sex and duration of mechanical ventilation aiming to recruit at least 100 participants. The measurements will be conducted at 6 months after ICU discharge.

\section{Family or next of kin interviews}

During the 3-month follow-up call, patients will be asked if a family member or next of kin will be willing to participate in the interview study. Once monthly, we will purposely select a sample of family members to be contacted. The selection will be performed to ensure maximum variation in terms of age, educational level, length of ICU stay, treatment centre, and COVID-19 status of the patient that went through ICU. Information about the interview study will be provided over the phone following a script approved by the ethics committee. Once the family members verbally consent, the interview will be scheduled. Interviews will be semistructured and be recorded for later transcription verbatim. The interviewer is a clinical psychologist with experience conducting interviews and training on providing emotional support for people under distress. We will aim to conduct 18 interviews or more until data saturation is achieved. Interviews will cover four main topic areas: ICU admission, communication during the ICU stay, experience of returning home and the experience of having a loved one in the ICU (online supplemental file 1). Each transcription will be anonymised, and the recording will be securely deleted.

\section{Critical care staff interviews}

Once the bed occupancy in ICU returns to usual levels, recruitment will start. An open call to participate will be made through WhatsApp and Facebook groups of the clinicians working in the participating centres. Additionally, posters will be put in the rest areas to capture a wider population. We will recruit medics, nurses, healthcare assistants and physiotherapists that normally work in an ICU and have patient-facing clinical duties for more than 96 hours during the pandemic. The invitation to participate will lead to a Google Form containing information about the study and a short script that constitutes the informed consent. From the list of volunteers, we will purposely sample three professionals per clinical group aiming to maximise variation regarding years of experience and centre where they work. We expect a minimum of 40 interviews, but we will continue recruitment until data saturation is achieved. Interviews will be conducted online or over the phone. Participants will be asked for verbal consent before starting the interview, which will be recorded for later transcription verbatim. Interviews will be semistructured covering five main topic areas: preparation before the pandemic; intellectual, physical, and emotional challenges during the pandemic; and learning for future events (online supplemental file 1).

\section{Follow-up feasibility}

The consent rate will be collected, calculating the number of patients who agreed to participate divided by the number of patients who meet selection criteria, expecting a consent rate $>70 \%{ }^{41}$ The feasibility overtime during the follow-up will be measured as cohort retention rate, considering the number of patients who can be contacted and evaluated at 3 months and 6 months, expecting a cohort retention rate $>70 \%$ as elsewhere. ${ }^{42-44}$ Additionally, the reasons for the lack of assessments will be recorded individually.

\section{Sample size calculation}

All patients meeting the eligibility criteria discharged from ICU between October 2020 and April 2021 (due to funding constraints) will be invited to participate. Based on bed capacity and patient flow from previous years, we estimated that 20-30 mechanically ventilated adult patients are discharged monthly from each centre. This 
means the sampling universe ranges from 840 to 1260 patients.

Hodgson $e t a l^{8}$ found that a quarter of ICU survivors had severe or moderate disability at 6 months after discharge, and half of them had mild disability. ${ }^{8}$ There is no information to estimate how much the prevalence of disability increases during a pandemic; however, the prevalence of mental health issues could be used as a proxy of the expected impact on physical health. Hodgson et $a l^{8}$ found that $22 \%$ of patients had anxiety or depressive symptoms at 6 months after discharge. Lee $e t a t^{55}$ found that among survivors of the SARS outbreak, $40 \%$ had at least moderate anxiety 1 year after. ${ }^{42}$ This is equivalent to a relative risk of 1.81 . Considering that measurement time points are different, we have estimated our sample size assuming a relative risk (RR) of 1.5 or 1.6 , which is more conservative than the estimation based on the literature. The different scenarios used for the sample size calculation appear in table 3 .

The most plausible scenario is that $40 \%$ of ICU survivors discharged in a low demand period will have some degree of disability, and this will increase to $64 \%$ for those discharged during high demand periods. Considering loss to follow-up, we estimate a total of 550 patients need to be recruited at ICU discharge, so 413 patients are assessed at 3 months after discharge (25\% loss to follow-up) and 289 patients at 6 months ( $30 \%$ lost to follow-up).

\section{Quantitative analysis}

Categorical variables will be presented as absolute and relative frequencies for each subgroup (ie, admission diagnosis and treatment centre) and time point (ie, ICU discharge, 3-month and 6-month follow-up). In the case of normally distributed continuous variables, these will be summarised using the mean and SD, while for those nonnormally distributed, the median and IQR will be used instead.

The trajectory for each outcome measure will be estimated using longitudinal multilevel regression with robust SEs to account for data coming from seven treatment centres. The comparison according to periods of low and high demand will be performed by adding an interaction term. All models will be adjusted for age, sex, duration of mechanical ventilation and the Charlson Comorbidity Index. If data have a normal distribution, a linear regression model will be chosen. In the case of right skewed data, a Poisson regression will be used. For HADS, IES-R and WHODAS 2.0, data will be analysed as total scores and categories given by each questionnaire. Longitudinal multilevel modelling is robust to missing values when these are missing at random. We will test for this assumption by comparing age, sex, duration of mechanical ventilation and the Charlson Comorbidity Index of patients loss to follow-up and those who were assessed at all time points. If the assumption is not met, we will use a regression model to estimate the score values of patients with similar characteristics regard the four variables aforementioned.

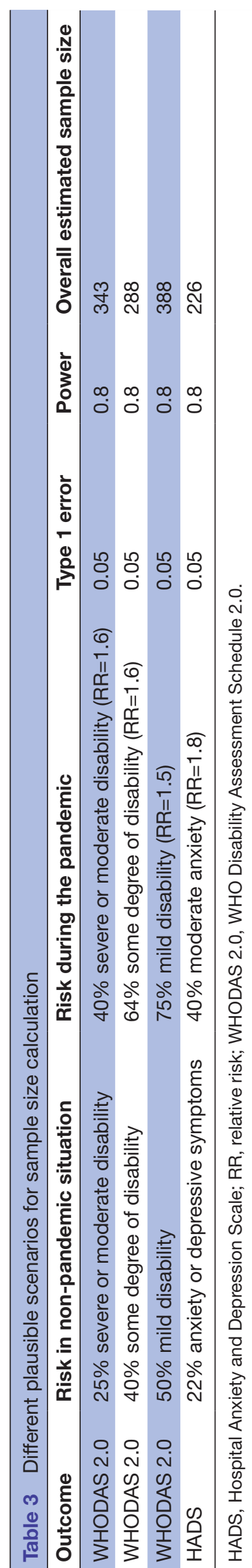


Survival will be analysed using Kaplan-Meier curves. If the assumption of proportional hazards is met, survival will be compared between patients admitted due to COVID-19 versus other causes using Cox regression. The Bonferroni correction for multiple testing will be used to adjust $\mathrm{p}$ values. All analyses will be performed in Stata V.16.0 SE.

\section{Qualitative analysis}

Data from the interviews with family members and critical care staff will be analysed using framework analysis. ${ }^{46}$ Transcription will be aided by the software Scrintal and analysis by NVivo V.12.0. Two coders will listen and read in-full all interviews before meeting to explore potential common topics that were discussed during the interviews. These topics will form the initial coding framework. Through an iterative process, these codes will be refined into overarching themes capturing differences and similarities across subgroups. A more advanced coding framework will be reviewed with members of the research team until agreement regarding the final framework is reached.

Themes will be used to explain the experience of family members during the pandemic and, potentially, identify areas where improvements could be made in the future. In the case of critical care staff, the aim is to explore to what extent the approach to the pandemic of each centre influenced the experience of the different clinical groups and what can be learnt for future outbreaks.

Findings will be shared with our participants and with other family members/critical care staff that did not participate in the interviews to ensure our interpretation reflects their experiences.

\section{Patient and public involvement}

Patients or the public were not involved in the design, conduct, reporting or dissemination plans of this study.

\section{DISCUSSION}

To the best of our knowledge, this is the first Chilean multicentre study assessing functional outcomes related to PICS in mechanically ventilated patients using the Core Outcome Measurement Set for critical illness survivors. The findings of this study will help determine the effect of the current pandemic in the prevalence of PICS in ICU survivors treated in public and private hospitals in Chile. This study will also explore experiences of family members/carers and healthcare professionals working during the current pandemic. Learnings regarding how to improve recruitment of participants, the practicalities of data collection during a pandemic, and strategies to reduce/prevent attrition will be helpful for future cohort studies in the country and elsewhere.

\section{Limitations}

This study is not exempt of limitations. First, the pandemic imposes conditions that we cannot control for. Localised lockdowns affected differently the participating hospitals; therefore, it could not be predicted whether the follow-up of these patients could include face-to-face assessments or not due to the risk of infection and legal restrictions. Additionally, leaves of absence due to sickness and being a close contact of a COVID-19 case meant that the skill mix and workload of the healthcare professionals varied widely in each unit and day to day.

Second and related to the uncertainty of conducting face-to-face measurements, we designed this study to include self-reported at 3 months and 6 months after ICU discharge. This means we were unable to perform the 6 min walk test, ${ }^{47}$ which is a recommended measure of physical functioning. Instead, we will collect self-reported measures such as the EQ-5D and WHODAS 2.0, which can be assessed over the phone. Additionally, we will assess in a subsample physical activity using actigraphy, which will provide valuable information about sedentary behaviours of ICU survivors. Third, the variables and assessments collected at baseline are a balance between building a comprehensive picture of the mental, physical, and cognitive state of each participant and what was a reasonable time commitment for the healthcare professionals working during the pandemic.

\section{Ethics and dissemination \\ Ethical considerations}

The IMPACCT COVID-19 study is conducted in accordance with the Declaration of Helsinki. Due to the observational nature of this study, patients will not be exposed to any intervention, just observing the evolution of outcomes from ICU discharge to 6 months after. This study was reviewed and approved by the Clínica Alemana Research and Clinical Trials Unit and the Facultad de Medicina Clínica Alemana Universidad del Desarrollo Ethics Committee (registration number 2020-78) and the Servicio de Salud Metropolitano Oriente Ethics Committee (registration number 152-0029). The protocol was also reviewed and approved by the clinical director of each participating ICU department. All recruited patients will be informed on the study obtaining their written informed consent before the first evaluation. Patients will receive verbal and written information related to postintensive care syndrome at the ICU discharge evaluation. At the 3-month or 6-month evaluation, patients with moderate or severe disability (according to the WHODAS 2.0 results) will receive information on rehabilitation alternatives at their nearest hospital.

\section{Dissemination}

We will disseminate results to key stakeholders including critical care clinicians, patients, families, rehabilitation staff, research funders and the public.

The knowledge translation of the IMPACCT COVID-19 study will follow the three end-of-grant knowledge translation strategy categories: diffusion (let it happen), dissemination (help it happen) and application (make it happen). ${ }^{48}$ Diffusion will be carried out using social media such as Twitter and ResearchGate. Dissemination will be carried 
out through presentation of findings in conference meetings and peer-review journal publications following the Strengthening the Reporting of Observational studies in Epidemiology guidelines. Additionally, the progress, preliminary findings and final results will be disseminated on the study's website (https://medicina.udd.cl/kinesiologia-santiago/impacct). Application will include workshops, academic meetings, and development of useful tool for the follow-up of ICU survivors for both clinicians and researchers.

\section{Author affiliations}

${ }^{1}$ School of Physical Therapy, Facultad de Medicina Clínica Alemana Universidad del Desarrollo, Santiago, Chile

${ }^{2}$ Department of Health Sciences, University of York, Heslington, UK

${ }^{3}$ Servicio de Medicina Física y Rehabilitación and Departamento de Paciente Crítico, Clinica Alemana Universidad del Desarrollo, Santiago, Chile

Twitter Ana Cristina Castro-Ávila @ac_castroavila, Catalina Merino-0sorio @ MerinoCatalina, Felipe González-Seguel @FGonzalezSeguel, Agustín Camus-Molina @acamusm and Jaime Leppe @jeleppe

Acknowledgements We would like to acknowledge the Universidad del Desarrollo for funding this research and each participating site for allowing us to use part of their infrastructure (organised alphabetically): Clínica Alemana de Santiago, Clínica BUPA, Clínica INDISA, Hospital del Salvador, Hospital Metropolitano, Hospital Regional Dr Leonardo Guzmán de Antofagasta, and Hospital San Pablo de Coquimbo.

Collaborators The IMPACCT COVID-19 study group includes (organised alphabetically): Ana Castro-Ávila, Agustín Camus-Molina, Catalina MerinoOsorio, Felipe González-Seguel, and Jaime Leppe; and the following additional collaborators: Camilo Cáceres Parra, Eduardo González Tapia, Felipe Muñoz-Muñoz, Fernanda Baus Auil, Javiera Aguilera Scarpati, Joaquín Olave, Macarena LeivaCorvalán, Pilar Castro, and Yerko Villagra Jofré.

Contributors All authors meet the ICMJE criteria for authorship.

Funding Impact on Mental, Physical, And Cognitive functioning of a Critical care sTay during the COVID-19 pandemic (IMPACCT COVID-19) study is funded by Universidad del Desarrollo (Grant number 2020-78) and sponsored by the Chilean National Agency for Research and Development (ANID-0772).

Disclaimer The funders had no role in the design, collection, analysis of the study and writing of this manuscript.

Competing interests None declared.

Patient consent for publication Not required.

Provenance and peer review Not commissioned; externally peer reviewed.

Supplemental material This content has been supplied by the author(s). It has not been vetted by BMJ Publishing Group Limited (BMJ) and may not have been peer-reviewed. Any opinions or recommendations discussed are solely those of the author(s) and are not endorsed by BMJ. BMJ disclaims all liability and responsibility arising from any reliance placed on the content. Where the content includes any translated material, BMJ does not warrant the accuracy and reliability of the translations (including but not limited to local regulations, clinical guidelines, terminology, drug names and drug dosages), and is not responsible for any error and/or omissions arising from translation and adaptation or otherwise.

Open access This is an open access article distributed in accordance with the Creative Commons Attribution Non Commercial (CC BY-NC 4.0) license, which permits others to distribute, remix, adapt, build upon this work non-commercially, and license their derivative works on different terms, provided the original work is properly cited, appropriate credit is given, any changes made indicated, and the use is non-commercial. See: http://creativecommons.org/licenses/by-nc/4.0/.

\section{ORCID iDs}

Ana Cristina Castro-Ávila http://orcid.org/0000-0003-4475-4325

Catalina Merino-0sorio http://orcid.org/0000-0002-6836-5466

Felipe González-Seguel http://orcid.org/0000-0001-9099-2389

Agustín Camus-Molina http://orcid.org/0000-0001-7378-9565

Jaime Leppe http://orcid.org/0000-0002-8663-1182
REFERENCES

1 Herridge MS, Tansey CM, Matté A, et al. Functional disability 5 years after acute respiratory distress syndrome. $N$ Engl J Med 2011;364:1293-304.

2 Pfoh ER, Wozniak AW, Colantuoni E, et al. Physical declines occurring after hospital discharge in ARDS survivors: a 5-year longitudinal study. Intensive Care Med 2016;42:1557-66.

3 Van Aerde N, Meersseman P, Debaveye Y, et al. Five-year impact of ICU-acquired neuromuscular complications: a prospective, observational study. Intensive Care Med 2020;46:1184-93.

4 van Sleeuwen D, van de Laar F, Geense W, et al. Health problems among family caregivers of former intensive care unit (ICU) patients: an interview study. BJGP Open 2020;4:bjgpopen20X101061.

5 Griffiths J, Hatch RA, Bishop J, et al. An exploration of social and economic outcome and associated health-related quality of life after critical illness in general intensive care unit survivors: a 12-month follow-up study. Crit Care 2013;17:R100.

6 Martillo MA, Dangayach NS, Tabacof L, et al. Postintensive care syndrome in survivors of critical illness related to coronavirus disease 2019: cohort study from a New York city critical care recovery clinic. Crit Care Med 2021;49:1427-38.

7 Huang C, Huang L, Wang Y, et al. 6-month consequences of COVID-19 in patients discharged from Hospital: a cohort study. Lancet 2021;397:220-32.

8 Hodgson CL, Udy AA, Bailey M, et al. The impact of disability in survivors of critical illness. Intensive Care Med 2017;43:992-1001.

9 Hatch R, Young D, Barber V, et al. Anxiety, depression and post traumatic stress disorder after critical illness: a UK-wide prospective cohort study. Crit Care 2018;22:310.

10 Pun BT, Badenes R, Heras La Calle G, et al. Prevalence and risk factors for delirium in critically ill patients with COVID-19 (COVID-D): a multicentre cohort study. Lancet Respir Med 2021;9:239-50.

11 Pearlin LI, Mullan JT, Semple SJ, et al. Caregiving and the stress process: an overview of concepts and their measures. Gerontologist 1990;30:583-94.

12 Braithwaite J. Changing how we think about healthcare improvement. BMJ 2018;361:k2014.

13 De Jonghe B, Sharshar T, Lefaucheur J-P, et al. Paresis acquired in the intensive care unit: a prospective multicenter study. JAMA 2002;288:2859-67.

14 NHS Digit. Organ system support, 2020. Available: https:// datadictionary.nhs.uk/attributes/organ_system_supported.html

15 Needham DM, Sepulveda KA, Dinglas VD, et al. Core outcome measures for clinical research in acute respiratory failure survivors. An international modified Delphi consensus study. Am J Respir Crit Care Med 2017;196:1122-30.

16 Turnbull AE, Sepulveda KA, Dinglas VD, et al. Core domains for clinical research in acute respiratory failure survivors. Crit Care Med 2017;45:1001-10.

17 World Health Organization. The International classification of functioning, disability and health (ICF). Geneva, Switzerland: World Health Organization, 2001.

18 Organización Mundial de la Salud (OMS). Medición de la Salud y la Discapacidad: manual para el cuestionario de evaluación de la discapacidad de la OMS: WHODAS 2.02015.

19 Rockwood K, Theou O. Using the clinical frailty scale in allocating scarce health care resources. Can Geriatr J 2020;23:254-9.

20 Nakajima H, Nishikimi M, Shimizu M, et al. Clinical frailty scale score before ICU admission is associated with mobility disability in septic patients receiving early rehabilitation. Crit Care Explor 2019;1:e0066.

21 Geriatric Medicine Research. Geriatric medicine research of the Department of medicine at Dalhousie university, 2020. Available: https://www.dal.ca/sites/gmr/our-tools/clinical-frailty-scale/cfsguidance.html

22 Vanpee G, Hermans G, Segers J, et al. Assessment of limb muscle strength in critically ill patients: a systematic review. Crit Care Med 2014;42:701-11.

23 Hermans G, Clerckx B, Vanhullebusch T, et al. Interobserver agreement of medical Research Council sum-score and handgrip strength in the intensive care unit. Muscle Nerve 2012;45:18-25.

24 Huang M, Chan KS, Zanni JM, et al. Functional status score for the ICU: an international Clinimetric analysis of validity, responsiveness, and minimal important difference. Crit Care Med 2016;44:e1155-64.

25 González-Seguel F, Camus-Molina A, Leppe J, et al. Chilean version of the functional status score for the intensive care unit: a translation and cross-cultural adaptation. Medwave 2019;19:e7439.

26 Camus-Molina A, González-Seguel F, Castro-Ávila AC, et al. Construct validity of the Chilean-Spanish version of the functional status score for the intensive care unit: a prospective observational study using actigraphy in mechanically ventilated patients. Arch Phys Med Rehabil 2020;101:1914-21. 
27 Stienen MN, Geisseler O, Velz J, et al. Influence of the intensive care unit environment on the reliability of the Montreal cognitive assessment. Front Neurol 2019;10:734

28 Delgado C, Araneda A, Behrens MI. Validación del instrumento Montreal cognitive assessment en español en adultos mayores de 60 años. Neurología 2019;34:376-85.

29 Bello-Lepe S, Alonso-Sánchez MF, Ortega A, et al. Montreal cognitive assessment as screening measure for mild and major neurocognitive disorder in a Chilean population. Dement Geriatr Cogn Dis Extra 2020;10:105-14.

30 Jutte JE, Needham DM, Pfoh ER, et al. Psychometric evaluation of the hospital anxiety and depression scale 3 months after acute lung injury. J Crit Care 2015;30:793-8.

31 Villoria E, Lara L. Assessment of the hospital anxiety and depression scale for cancer patients. Rev Med Chil 2018;146:300-7.

32 Hosey MM, Bienvenu OJ, Dinglas VD, et al. The IES-R remains a core outcome measure for PTSD in critical illness survivorship research. Crit Care 2019;23:362.

33 Caamaño W L, Fuentes M D, González B L, et al. Adaptación Y validación de la versión chilena de la escala de impacto de eventorevisada (EIE-R). Rev Med Chil 2011;139:1163-8.

34 Oeyen SG, Vandijck DM, Benoit DD, et al. Quality of life after intensive care: a systematic review of the literature. Crit Care Med 2010;38:2386-400.

35 EuroQol - a new facility for the measurement of health-related quality of life. Health Policy 1990;16:199-208.

36 Zarate V, Kind P, Valenzuela P, et al. Social valuation of EQ-5D health states: the Chilean case. Value Health 2011;14:1135-41.

37 Norman BC, Jackson JC, Graves JA, et al. Employment outcomes after critical illness. Crit Care Med 2016;44:2003-9.

38 Kamdar BB, Suri R, Suchyta MR, et al. Return to work after critical illness: a systematic review and meta-analysis. Thorax 2020;75:17-27.
39 Leppe J, Munizaga B, Margozzini P. Physical activity and sedentary behavior according National health survey 2016-17. Medwave 2019;19:SP74.

40 Aguilar-Farias N, Leppe Zamora J. Is a single question of the global physical activity questionnaire (GPAQ) valid for measuring sedentary behaviour in the Chilean population? J Sports Sci 2017;35:1-6.

41 Kho ME, Molloy AJ, Clarke FJ, et al. TryCYCLE: a prospective study of the safety and feasibility of early In-Bed cycling in mechanically ventilated patients. PLoS One 2016;11:e0167561.

42 Nunna K, Al-Ani A, Nikooie R, et al. Participant retention in followup studies of acute respiratory failure survivors. Respir Care 2020;65:1382-91.

43 Mehrholz J, Mückel S, Oehmichen F, et al. First results about recovery of walking function in patients with intensive care unitacquired muscle weakness from the general weakness syndrome therapy (GymNAST) cohort study. BMJ Open 2015;5:e008828.

44 Thomas S, Mehrholz J. Health-related quality of life, participation, and physical and cognitive function of patients with intensive care unit-acquired muscle weakness 1 year after rehabilitation in Germany: the GymNAST cohort study. BMJ Open 2018;8:e020163.

45 Lee AM, Wong JGWS, McAlonan GM, et al. Stress and psychological distress among SARS survivors 1 year after the outbreak. Can $J$ Psychiatry 2007;52:233-40.

46 Braun V, Clarke V. Using thematic analysis in psychology. Qual Res Psychol 2006;3:77-101.

47 Haines KJ, Hibbert E, McPeake J, et al. Prediction models for physical, cognitive, and mental health impairments after critical illness: a systematic review and critical appraisal. Crit Care Med 2020;48:1871-80.

48 Canadian Institutes of Health Research. Guide to knowledge translation planning at CIHR: integrated and End-of-grant approaches, 2012. Available: www.cihr-irsc.gc.ca/e/45321.htm 